\title{
ON WHEN A GRADED RING IS GRADED EQUIVALENT TO A CROSSED PRODUCT
}

\author{
JEREMY HAEFNER
}

(Communicated by Ken Goodearl)

\begin{abstract}
Let $R$ be a ring graded by a group $G$. We are concerned with describing those $G$-graded rings that are graded equivalent to $G$-crossed products. We give necessary and sufficient conditions for when a strongly graded ring is graded equivalent to a crossed product, provided that the 1-component is either Azumaya or semiperfect. Our result uses the torsion product theorem of Bass and Guralnick. We also construct various examples of such rings.
\end{abstract}

\section{INTRODUCTION}

In this paper, all rings have an identity element. Let $R$ and $S$ be rings graded by a group $G$. Following [12], [16], [13], and [14], we say that $R$ and $S$ are graded equivalent provided there is a graded $R$-module $P$ such that $P$ is an $R$-progenerator and $\operatorname{End}_{R}(P) \cong S$ as graded rings. This paper considers the following problem:

Describe those $G$-graded rings that are graded equivalent to $G$-crossed products.

The significance of working with a $G$-graded ring that is graded equivalent to a $G$-crossed product is that graded equivalence, in a certain sense, "preserves" the categorical structure of the crossed product. For example, if $R$ is strongly $G$-graded and graded equivalent to a $G$-crossed product $S$, then not only are the categories $R$-mod and $S$-mod equivalent, but so are the categories $R_{H}$-mod and $S_{H}$-mod for each subgroup $H$ of $G$. ( $R_{H}$ denotes the truncation of $R$ at the subgroup $H$.) See [14].

The Cohen-Montgomery duality theory is an important application of how problems about graded rings can be reduced to problems about crossed products via graded equivalence. Briefly, if $R$ is a ring graded by a finite group $G$, Cohen and Montgomery construct a ring $R \# G$ (the smash product of $R$ by $G$ ) in such a way that $G$ acts as automorphisms on $R \# G$. If $P=\coprod_{g \in G} R(g)$, where $R(g)=R$ as $R$-modules but is graded via $R(g)_{h}=R_{g h}$, then $P$ is a graded $R$-module and an $R$-progenerator such that $\operatorname{End}_{R}(P)$ and the skew group ring $(R \# G) * G$ are isomorphic as graded rings ([18]). Consequently, if $G$ is finite, a $G$-graded $\operatorname{ring} R$ is always graded equivalent to the skew group $\operatorname{ring}(R \# G) * G$. On the other hand, if $G$ is infinite, then $R$ may not be graded equivalent to a crossed product ([15]). In light of this, the problem posed above may be rephrased as describing those

Received by the editors April 26, 1994 and, in revised form, September 6, 1994.

1991 Mathematics Subject Classification. Primary 16D90, 16S35, 16S40, 16S50, 16W50.

The author's research was partially supported by the National Security Agency. 
$G$-graded rings that are graded equivalent to $G$-crossed products, when the group $G$ is infinite.

Since Menini and Năstăsescu have determined when a $G$-graded ring is graded equivalent to a $G$-strongly graded ring ([16, Theorem 2.2$])$, and since any crossed product is strongly graded, we assume, for the remainder of this paper, that $R$ is strongly graded. We show, in Theorem 3, that any $G$-strongly graded ring $R$ such that the 1-component is semiperfect is graded equivalent to a crossed product. Our main result, however, is Theorem 10, which provides necessary and sufficient conditions for when a strongly graded ring is graded equivalent to a crossed product, whenever the 1-component is commutative, Noetherian with Krull dimension $d$. We extend this result, in Corollary 14, by requiring only that the 1-component be an Azumaya algebra. To illustrate these theorems, we provide various examples in $\S 5$.

As a last introductory remark, if we were to consider rings without identity elements and appropriately alter the definition of graded equivalence, then every $G$-graded ring $R$ is graded equivalent to a skew group $\operatorname{ring} T * G$, where $T$ need not have an identity element. See [6], [18], [13], and [14]. In contrast, our requirement for this paper is to work only with rings with identity.

We thank M. Beattie for her helpful comments in the writing of this paper.

\section{DEFINITIONS AND PRELIMINARIES}

Throughout this paper, all 1-sided modules are left modules and we write module homomorphisms on the right. Ring and group homomorphisms, however, are written on the left.

Definition 1. If $T$ is any ring, let $T^{\bullet}$ denote the units of $T, \operatorname{Pic}(T)$ denote the set of isomorphism classes of invertible $T$-bimodules and Picent $(T)$ denote the set of isomorphism classes of invertible bimodules that are centralized by the center of $T$. Following [11], let $\mathrm{Pic}_{n}(T)$ denote the isomorphism classes of invertible $T$ bimodules $P$ for which there is a left isomorphism $P^{(n)} \cong T^{(n)}$. (In this paper, the notation $X^{(n)}$ refers to the direct sum of $n$ copies of $X$.) Analogously, we define $\operatorname{Picent}_{n}(T)$.

For any $T$-bimodule $X$ and for any $\sigma \in \operatorname{Aut}(T)$, we define a new bimodule ${ }_{1}(X)_{\sigma}$. As a left module, ${ }_{1}(X)_{\sigma}=X$, while the right $T$-action is given by $x \cdot t=x \sigma(t)$. In particular, this definition induces a group homomorphism $\omega: \operatorname{Aut}(T) \rightarrow \operatorname{Pic}(T)$ which is defined via $\omega: \sigma \rightarrow{ }_{1}(T)_{\sigma}$. See [8, Theorem 55.11] for further details.

Finally, $R$ denotes a strongly $G$-graded ring, which we write as $R=\bigoplus_{g \in G} R_{g}$. Recall that a crossed product is a $G$-graded ring $R$ such that each component $R_{g}$ contains a unit $u_{g}$ of the ring $R$ ([17, Example 2, p. 18]), a skew group ring is a $G$-crossed product such that the set of units $\left\{u_{g} \mid g \in G\right\}$ forms a group and is isomorphic to $G$, and a twisted group ring $R$ is a crossed product ring such that each component contains a unit of $R$ that commutes with $R_{1}$. Since $R$ is strongly graded, we define the component group of $R, \operatorname{Comp}(R)$, to be the subgroup of $\operatorname{Pic}\left(R_{1}\right)$ consisting of the bimodule isomorphism classes of the components $R_{g}$ of $R$.

Lemma 2. Let $R$ be $G$-strongly graded.

(1) $R_{g} \cong R_{1}$ as left modules $\Leftrightarrow R_{g}$ contains a unit of $R \Leftrightarrow R_{g} \cong{ }_{1}\left(R_{1}\right)_{\theta}$ (as $R_{1}$-bimodules) for some $\theta \in \operatorname{Aut}\left(R_{1}\right) \Leftrightarrow R_{g} \in \operatorname{im} \omega$. 
(2) $R$ is a crossed product $\Leftrightarrow \operatorname{Comp}(R) \subset \operatorname{im} \omega \Leftrightarrow$ there exists a map $\Theta: G \rightarrow R^{\bullet}$ such that $R_{g}=R_{1} \cdot \Theta(g)=\Theta(g) \cdot R_{1} \Leftrightarrow$ there exists a map $\Delta: G \rightarrow \operatorname{Aut}\left(R_{1}\right)$ such that $R_{g} \cong{ }_{1}\left(R_{1}\right)_{\Delta(g)}$ as bimodules.

(3) $R$ is a twisted group ring $\Leftrightarrow \operatorname{Comp}(R)=1$ (as a subgroup of $\operatorname{Pic}\left(R_{1}\right)$ ) $\Leftrightarrow$ there exists a map $\Theta: G \rightarrow R^{\bullet}$ such that $R_{g}=R_{1} \cdot \Theta(g)$ and $\Theta(g)$ commutes with the elements of $R_{1}$.

(4) $R$ is a skew group ring $\Leftrightarrow$ there exists a group homomorphism $\Theta: G \rightarrow R^{\bullet}$ such that $R_{g}=R_{1} \cdot \Theta(g)=\Theta(g) \cdot R_{1} \Leftrightarrow$ there exists a group homomorphism $\Delta: G \rightarrow \operatorname{Aut}\left(R_{1}\right)$ such that $R_{g} \cong{ }_{1}\left(R_{1}\right)_{\Delta(g)}$ as bimodules.

(5) (Dade) $R$ is graded equivalent to a crossed product $\Leftrightarrow$ there is a progenerator $P$ of $\bmod \left(R_{1}\right)$ such that $P$ is 'fixed' under the tensor action of $\operatorname{Comp}(R)$ (i.e., $R_{g} \otimes P \cong P$ for every $\left.g \in G\right)$.

Proof. (1) This is a special case of [8, Theorem 55.12].

(2) $R$ is a crossed product if and only if each component $R_{g}$ contains a unit $u_{g}$ of $R$. Now apply (1).

(3) $R$ is a twisted group ring if and only if each component $R_{g}$ contains a unit $u_{g}$ of $R$ and $u_{g}$ commutes with the elements of $R_{1}$. Thus, $R$ is a twisted group ring $\Leftrightarrow$ each $R_{g} \cong R_{1}$ as bimodules $\Leftrightarrow \operatorname{Comp}(R)=1$.

(4) $R$ is a skew group ring if and only if each component $R_{g}$ contains a unit $u_{g}$ of $R$ and the set of these units $\left\{u_{g} \mid g \in G\right\}$ forms a group isomorphic to $G$.

(5) This is [9, Corollary 5.14].

The above lemma affords us an opportunity to solve the problem posed in the Introduction when $R_{1}$ is semiperfect.

Theorem 3 (The semiperfect case). If $R$ is a $G$-strongly graded ring such that $R_{1}$ is semiperfect, then $R$ is graded equivalent to a $G$-crossed product $S$. Moreover, $S_{1}$ is the basic ring of $R_{1}$.

Proof. Set $T=R_{1}$. Using [2, Proposition 27.10], there exists a set $\left\{e_{1}, \ldots, e_{m}\right\}$ of pairwise orthogonal idempotents such that $T e_{1}, \ldots, T e_{m}$ is a complete irredundant set of representatives of the primitive left $T$-modules. In particular, if $X$ is a projective, indecomposable, left $T$-module, then $X \cong T e_{i}$ for some $i$. Set $Q=$ $\bigoplus_{i=1}^{m} T e_{i}$. Then $Q$ is a left $T$-progenerator. If $P \in \operatorname{Pic}(T)$, then $P \otimes T e_{i}$ is a projective indecomposable since $P^{-1} \otimes\left(P \otimes T e_{i}\right) \cong T e_{i}$. Moreover, $P \otimes T e_{i} \cong$ $P \otimes T e_{j} \Leftrightarrow i=j$. Consequently, $P \otimes Q \cong \bigoplus_{i=1}^{m}\left(P \otimes T e_{i}\right) \cong \bigoplus_{i=1}^{m} T e_{i}=Q$. In particular, $R_{g} \otimes Q \cong Q$ for every $g \in G$. Thus, by Lemma $2(5), R$ is graded equivalent to a crossed product $S=\operatorname{End}_{R}(R \otimes Q)$ where $S_{1}=\operatorname{End}_{T}(Q)$. It follows that $S_{g} \cong \operatorname{Hom}_{T}\left(Q, R_{g} \otimes Q\right)$.

\section{MATRIX RINGS}

We begin this section with an important but easy example of graded equivalence.

Example 4 ( $R$ and $M_{n}(R)$ are graded equivalent). Let $R$ be any $G$-graded ring. For any positive integer $n, M_{n}(R)$ is also $G$-graded with the grading defined by $M_{n}(R)_{g}=M_{n}\left(R_{g}\right)$. If $P$ is the free $R$-module of rank $n$ with the induced grading, it follows that $P$ is a graded, $R$-progenerator such that $\operatorname{End}_{R}(P) \cong M_{n}(R)$. Thus, $R$ and $M_{n}(R)$ are $G$-graded equivalent. 
Using the above example and Lemma 2, we can now prove the following key proposition that characterizes when a strongly graded $\operatorname{ring} R$ is graded equivalent to a crossed product of the form $M_{n}(R)$.

Theorem 5. The following statements are equivalent:

(1) There is a positive integer $n$ such that $R$ is graded equivalent to a crossed product of the form $M_{n}(R)$, which is graded via $M_{n}(R)_{g}=M_{n}\left(R_{g}\right)$.

(2) $\operatorname{Comp}(R) \subset \operatorname{Pic}_{n}\left(R_{1}\right)$ for some positive integer $n$.

(3) There exists a positive integer $n$ such that, for each $g \in G$, there exists an isomorphism $\mu_{g}: R_{1}^{(n)} \rightarrow R_{g}^{(n)}$ as left modules.

(4) There exist a positive integer $n$ and a function $\Theta: G \rightarrow\left(M_{n}(R)\right)^{\bullet}$ such that $M_{n}\left(R_{g}\right)=M_{n}\left(R_{1}\right) \cdot \Theta(g)=\Theta(g) \cdot M_{n}\left(R_{1}\right)$.

(5) There exist a positive integer $n$ and a function $\Delta: G \rightarrow \operatorname{Aut}\left(M_{n}\left(R_{1}\right)\right)$ such that $M_{n}\left(R_{g}\right) \cong{ }_{1}\left(M_{n}\left(R_{1}\right)\right)_{\Delta(g)}$ as bimodules.

Proof. First observe that statements (1), (4), and (5) are equivalent by Lemma 2 and Example 4. Statements (2) and (3) are equivalent by Definition 1. It suffices to show that (2) and (4) are equivalent.

$(2) \Rightarrow(4)$ Since $\operatorname{Comp}(R) \subset \operatorname{Pic}_{n}\left(R_{1}\right)$, there is, for each $g \in G$, a left module isomorphism $\Theta(g): R_{1}^{(n)} \rightarrow R_{g}^{(n)}$. If we identify $\operatorname{Hom}_{R_{1}}\left(R_{1}, R_{g}\right)$ with $R_{g}$, we may identify $\Theta(g)$ as an element of $M_{n}\left(R_{g}\right)$. We have that $M_{n}\left(R_{1}\right) \cdot \Theta(g) \subset M_{n}\left(R_{g}\right)$ and $M_{n}\left(R_{g}\right) \cdot \Theta(g)^{-1} \subset M_{n}\left(R_{1}\right)$, which yields $M_{n}\left(R_{1}\right) \cdot \Theta(g)=M_{n}\left(R_{g}\right)$. Thus, (4) holds by Lemma 2 .

$(4) \Rightarrow(2)$ Since $M_{n}\left(R_{g}\right)=M_{n}\left(R_{1}\right) \Theta(g), M_{n}\left(R_{g}\right) \in \operatorname{Pic}_{1}\left(M_{n}\left(R_{1}\right)\right)$ by Lemma 2. But, by [10, Lemma 3.1], $\operatorname{Pic}_{1}\left(M_{n}\left(R_{1}\right)\right) \cong \operatorname{Pic}_{n}\left(R_{1}\right)$ via the mapping $M_{n}(X) \mapsto X$. It follows that $R_{g} \in \operatorname{Pic}_{n}\left(R_{1}\right)$ and so $\operatorname{Comp}(R) \subset \operatorname{Pic}_{n}\left(R_{1}\right)$.

The set of units $\{\Theta(g) \mid g \in G\}$ in $M_{n}(R)$ that appear in the proof of Theorem 5 need not be a group under multiplication; this is precisely the obstruction preventing $M_{n}(R)$ from being a skew group ring.

Theorem 6. The following statements are equivalent:

(1) There is a positive integer $n$ such that $R$ is graded equivalent to a skew group ring $M_{n}(R)$ with grading $M_{n}(R)=\bigoplus_{g \in G} M_{n}\left(R_{g}\right)$.

(2) $\operatorname{Comp}(R) \subset \operatorname{Pic}_{n}\left(R_{1}\right)$ for some positive integer $n$ and the map $\Theta: G \rightarrow$ $M_{n}(R)^{\bullet}($ from Theorem 5$)$ is a group homomorphism.

(3) $\operatorname{Comp}(R) \subset \operatorname{Pic}_{n}\left(R_{1}\right)$ for some positive integer $n$ and the map $\Delta: G \rightarrow$ $\operatorname{Aut}\left(M_{n}(T)\right.$ ) (from Theorem 5) is a group homomorphism.

Proof. We first observe that (2) and (3) are equivalent by Lemma 2.

$(2) \Rightarrow(1)$ Since $\operatorname{Comp}(R) \subset \operatorname{Pic}_{n}\left(R_{1}\right)$ for some positive integer $n, R$ is graded equivalent to a crossed product ring of the form $M_{n}(R)$ by Theorem 5 . Moreover, there is a map $\Theta: G \rightarrow M_{n}(R)^{\bullet}$ such that $M_{n}\left(R_{g}\right)=M_{n}\left(R_{1}\right) \cdot \Theta(g)$. If $\Theta$ is a group homomorphism, then it follows that the 2-cocycle $\tau: G \times G \rightarrow M_{n}\left(R_{1}\right)^{\bullet}$ that defines the crossed product structure of $M_{n}(R)$ is trivial and so $M_{n}(R)$ is a skew group ring. See $[17$, p. 3$]$.

$(1) \Rightarrow(2)$ If $R$ is graded equivalent to a skew group ring of the form $M_{n}(R)$, then, by Theorem $5, \operatorname{Comp}(R) \subset \operatorname{Pic}_{n}(R)$. Since $M_{n}(R)$ is a skew group ring with grading given by $M_{n}(R)_{g}=M_{n}\left(R_{g}\right)$, it follows that the 2-cocycle $\tau: G \times G \rightarrow M_{n}\left(R_{1}\right) \bullet$ is trivial and so $\Theta(g) \cdot \Theta(h)=\Theta(g h)$ for each $g, h \in G$. Consequently, the map $\Theta$ is a group homomorphism. 
In contrast to the result above, a strongly graded $\operatorname{ring} R$ is graded equivalent to a twisted group ring of the form $M_{n}(R)$ with grading defined by $M_{n}(R)_{g}=M_{n}\left(R_{g}\right)$ if and only if $R$ itself is a twisted group ring.

Theorem 7. The following statements are equivalent:

(1) There is a positive integer $n$ such that $R$ is graded equivalent to a twisted group ring $M_{n}(R)$, with grading defined by $M_{n}(R)_{g}=M_{n}\left(R_{g}\right)$.

(2) $R$ is a twisted group ring.

(3) For every positive integer $n, M_{n}(R)$ is a twisted group ring with grading defined by $M_{n}(R)_{g}=M_{n}\left(R_{g}\right)$.

Proof. It is clear that $(2) \Rightarrow(1)$ and it is not difficult to see that $(2) \Leftrightarrow(3)$. Thus, it suffices to prove $(1) \Rightarrow(2)$.

If $M_{n}(R)$ is a twisted group ring, then, by Lemma 2 , each $M_{n}\left(R_{g}\right) \cong M_{n}\left(R_{1}\right)$ as $M_{n}\left(R_{1}\right)$-bimodules. Thus, for each $g \in G$, there is a linear isomorphism $f_{g}$ : $M_{n}\left(R_{1}\right) \rightarrow M_{n}\left(R_{g}\right)$ that commutes with all matrices from $M_{n}\left(R_{1}\right)$. Using the idempotent matrix $E$ which has 1 in the (1,1)-entry and zeros elsewhere, it follows that $f_{g}$ induces a $R_{1}$-bimodule isomorphism between $R_{1}$ and $R_{g}$. Since this holds for each $g \in G$, we see that each $R_{g} \cong R_{1}$ as $T$-bimodules. Consequently, $R$ is a twisted group ring by Lemma 2 .

Finally, combining Theorems 6 and 7, we get the following corollary:

Corollary 8. $R$ is graded equivalent to a group ring of the form $M_{n}(R)$ with grading defined by $M_{n}(R)_{g}=M_{n}\left(R_{g}\right)$ if and only if $R$ is a twisted group ring and the map $\Theta: G \rightarrow M_{n}(R)^{\bullet}$ from Theorem 5 is a group homomorphism.

\section{The Commutative And the locally free CASES}

Let $G$ be a group, let $T$ be a commutative, Noetherian ring of Krull dimension $d$, and let $R$ denote a ring strongly graded by $G$, whose 1 -component is $T$ (i.e., $\left.T=R_{1}\right)$.

Remark 9. (1) There is a split exact sequence

$$
1 \rightarrow \operatorname{Picent}(T) \rightarrow \operatorname{Pic}(T) \stackrel{\Phi}{\rightarrow} \operatorname{Aut}(T) \rightarrow 1
$$

where $\Phi$ is given by $M \mapsto \Phi_{M}$ and $\Phi_{M}$ is an automorphism of $T$ defined by the relation $t m=m \Phi_{M}(t)$ for all $m \in M$ and $t \in T$. The back map is $\omega: \operatorname{Aut}(T) \rightarrow$ $\operatorname{Pic}(T)$, defined in Definition 1. Thus, $\operatorname{Pic}(T)$ is the semi-direct product of Picent $(T)$ and $\operatorname{Aut}(T)$ ([8, Theorem 55.13]). In particular, for each $P \in \operatorname{Pic}(T)$, there is a unique element $\Phi_{P} \in \operatorname{Aut}(T)$ and a unique element $Q(P) \in \operatorname{Picent}(T)$ such that $P={ }_{1}(Q)_{\Phi_{P}}$. It is easy to see that $Q(P)={ }_{1}(P)_{\Phi_{P}^{-1}}$. See $[8, \S 55]$ for more details. Let $\mathcal{Q}: \operatorname{Pic}(T) \rightarrow \operatorname{Picent}(T)$ denote the projection of $\operatorname{Pic}(T)$ into $\operatorname{Picent}(T)$; i.e., $\mathcal{Q}(P)={ }_{1}(P)_{\Phi_{P}^{-1}}$. Note that $\mathcal{Q}$ need not be a group homomorphism.

(2) Let $\operatorname{Compcent}(R)$ denote the subgroup generated by the image of $\operatorname{Comp}(R)$ inside Picent $(T)$ under the projection map $\mathcal{Q}: \operatorname{Pic}(T) \rightarrow \operatorname{Picent}(T)$. Observe that since Compcent $(R)$ is contained in the abelian group $\operatorname{Picent}(T)$, Compcent $(R)$ has a finite torsion exponent if and only if the set $\mathcal{Q}(\operatorname{Comp}(R))$ has a finite torsion exponent.

The crux of the proof of the following theorem is a result of Bass and Guralnick, which connects the multiplicative structure of $\operatorname{Pic}\left(R_{1}\right)$ with the additive structure 
of $\mathbf{K}_{0}\left(R_{1}\right)$ ([4] and [5]). The work of Bass and Guralnick, in turn, heavily depends on some nontrivial $\mathbf{K}$-theoretical results of Bass. In particular, Bass proves in [3, Corollary 4.5, p. 476] that if $T$ is a commutative, Noetherian ring of Krull dimension $d$ and $P$ is a faithful, finitely generated, projective $T$-module such that $l$ is the least common multiple of its local ranks, then there is a (finitely generated) projective $T$-module $Q$ such that $P \otimes Q \cong T^{l^{d+1}}$. For example, if $P \in \operatorname{Pic}(T)$, then $l=1$ and $Q$ is simply the multiplicative inverse of $P$ in $\operatorname{Pic}(T)$.

Theorem 10. The following statements are equivalent:

(1) The ring $R$ is graded equivalent to a crossed product.

(2) The group $\operatorname{Compcent}(R)$ has a torsion exponent.

(3) $\operatorname{Comp}(R) \subset \operatorname{Pic}_{n}(T)$ for some integer $n$.

(4) There is a positive integer $n$ such that $M_{n}(R)$ is a crossed product with grading defined by $M_{n}(R)_{g}=M_{n}\left(R_{g}\right)$ and $R$ and $M_{n}(R)$ are graded equivalent.

Proof. First observe that statements (3) and (4) are equivalent by Theorem 5 . Clearly, $(4) \Rightarrow(1)$ and so it suffices to show $(1) \Rightarrow(2) \Rightarrow(3)$.

$(1) \Rightarrow(2)$ Since $R$ is strongly graded, we know, by Lemma 2 (statement (5)), that there is a left progenerator $P_{1}$ for $\bmod (T)$ such that $R_{g} \otimes_{T} P_{1} \cong P_{1}$ for all $g \in G$. Let $l$ denote the least common multiple of the local ranks of $P_{1}$ and set $n=l^{d+1}$, where $d$ is the Krull dimension of $T$. By [3, Corollary 4.5, p. 476] and the fact that $P_{1}$ is faithful, there is a projective $T$-module $S^{\prime}$ such that $P_{1} \otimes S^{\prime} \cong T^{(n)}$. Fix $g \in G$. Since $T \otimes P_{1} \cong R_{g} \otimes P_{1}$, it follows that $T \otimes P_{1} \otimes S^{\prime} \cong R_{g} \otimes P_{1} \otimes S^{\prime}$ and so $T^{(n)} \cong R_{g}^{(n)}$, as left modules. Since $\mathcal{Q}\left(R_{g}\right)={ }_{1}\left(R_{g}\right)_{\Phi_{R_{g}^{-1}}}$, we see that, as left modules, $\mathcal{Q}\left(R_{g}\right)$ and $R_{g}$ are isomorphic. Consequently, $T^{(n)} \cong \mathcal{Q}\left(R_{g}\right)^{(n)}$, as left modules. Taking $n$th exterior products, we see that $\otimes^{n} \mathcal{Q}\left(R_{g}\right) \cong R$ and so $n$ is a torsion exponent for Compcent $(R)$.

$(2) \Rightarrow(3)$ Assume that $\operatorname{Compcent}(R)$ has torsion exponent $e$ and set $n=e^{d+1}$. Fix $g \in G$ and set $I=\mathcal{Q}\left(R_{g}\right)$. By [5, Theorem 1], there exists a commutative extension ring $S$ of $T$ such that $I \otimes_{T} S \cong S$ (as $S$-modules) and $S$ is $T$-projective of rank $e$. By [3, Corollary 4.5, p. 476], there exists a projective $T$-module $Q$ such that $S \otimes_{T} Q \cong T^{(n)}$. Thus,

$$
T^{(n)} \cong S \otimes_{T} Q \cong\left(I \otimes_{T} S\right) \otimes_{T} Q \cong I \otimes_{T}\left(S \otimes_{T} Q\right) \cong I \otimes_{T} T^{(n)} \cong I^{(n)} .
$$

However, $R_{g} \cong \mathcal{Q}\left(R_{g}\right)$ as left modules (as we have seen above) and so we have $R_{g}^{(n)} \cong T^{(n)}$. Thus, $\operatorname{Comp}(R) \subset \operatorname{Pic}_{n}(T)$.

Note that if $G$ has a torsion exponent $e$, then so does $\operatorname{Comp}(R)$, and so $R$ is graded equivalent to a crossed product of the form $M_{n}(R)$, where $n$ can be taken to be $e^{d+1}$. In particular, if $G$ is finite, then $R$ is graded equivalent to a crossed product of the form $M_{|G|^{d+1}}(R)$. Of course, by the Cohen-Montgomery result mentioned in the Introduction and [18], $R$ is also graded equivalent to $M_{|G|}(R)$ with a special grading.

We also observe that if $R_{1}$ belongs to the center of $R$, then $\operatorname{Comp}(R)=$ Compcent $(R)$.

Corollary 11. If $R_{1}$ belongs to the center of $R$, then $R$ is graded equivalent to a crossed product if and only if $\operatorname{Comp}(R)$ has a torsion exponent. 
We turn our attention to a noncommutative version of Theorem 10 . We shall require that there is a bijection between $\operatorname{Comp}(R)$ and a subgroup of Picent of the center of the 1-component.

Definition 12. Let $R_{1}$ be an arbitrary ring, let $C$ denote the center of $R_{1}$ and assume $C$ is Noetherian of Krull dimension $d$. Following [11], if $\operatorname{Div}\left(R_{1}\right)$ denotes the category of isomorphism classes of bimodule direct summands of some number of copies of $R_{1}$, then there is a categorical bijection between $\operatorname{Div}\left(R_{1}\right)$ and the category $\operatorname{Proj}(C)$ of finitely generated projective $C$-modules. Let $F: \operatorname{Div}\left(R_{1}\right) \rightarrow \operatorname{Proj}(C)$ denote this bijection. Set $\operatorname{DPic}\left(R_{1}\right)=\operatorname{Div}\left(R_{1}\right) \cap \operatorname{Pic}\left(R_{1}\right)$, which is a subset of $\operatorname{Picent}\left(R_{1}\right)$ (since the elements of $\operatorname{Div}\left(R_{1}\right)$ are centralized by the center of $\left.R_{1}\right)$. By the above correspondence, there is a categorical bijection $F: \operatorname{DPic}\left(R_{1}\right) \rightarrow$ Picent $(C), F$ preserves direct summands and tensor products (see [11, p. 927]), and $F\left(R_{1}\right)=C$.

Theorem 13. If each $R_{g} \in \operatorname{DPic}\left(R_{1}\right)$, then the ring $R$ is graded equivalent to a crossed product if and only if $\operatorname{Comp}(R)$ has a torsion exponent.

Proof. Since each $R_{g} \in \operatorname{DPic}\left(R_{1}\right)$, it follows that $\operatorname{Comp}(R) \subset \operatorname{Picent}\left(R_{1}\right)$ and $\operatorname{Comp}(R)=\operatorname{Compcent}(R)$. Suppose $R$ is graded equivalent to a crossed product. As before, there exists a progenerator $P_{1}$ for $R_{1}$ such that $R_{g} \otimes_{R_{1}} P_{1} \cong P_{1}$ for all $g \in G$. Applying the categorical bijection $F$ mentioned in Definition 12 , we see that $F\left(R_{g}\right) \otimes_{C} F\left(P_{1}\right) \cong F\left(P_{1}\right)$ for all $g \in G$. Now by the proof of Theorem 10, there is a finite torsion exponent for the set $\left\{F\left(R_{g}\right) \mid g \in G\right\}$ and hence, $\operatorname{Comp}(R)$ also has a finite exponent.

Conversely, if $\operatorname{Comp}(R)$ has a finite torsion exponent, then so does $F(\operatorname{Comp}(R))$. Invoking the proof of Theorem 10, there exists an integer $n$ such that $F\left(R_{g}\right) \otimes C^{n} \cong$ $C^{n}$ for all $g \in G$. Since $F\left(R_{1}\right)=C$, it follows that $R_{1}^{n}$ is a progenerator for $R_{1}$ such that $R_{g} \otimes R_{1}^{n} \cong R_{1}^{n}$ for all $g \in G$. Hence, $R$ is graded equivalent to a crossed product by Lemma 2 .

Corollary 14. Let $R$ be a ring strongly graded by $G$ such that $R_{1}$ is an Azumaya algebra of degree $m$ with center $C$. Assume each $R_{g}$ is centralized by the center of $R$. Then $R$ is graded equivalent to a crossed product if and only if $\operatorname{Comp}(R)$ has a torsion exponent.

Proof. By hypothesis, $\operatorname{Comp}(R) \subset \operatorname{Picent}\left(R_{1}\right)$. By [10, Proposition 4.1], $\operatorname{DPic}\left(R_{1}\right)$ $=\operatorname{Picent}\left(R_{1}\right) \cong \operatorname{Picent}(C)$. Now apply Theorem 13 .

\section{EXAMPLES}

We construct various examples of graded rings where the group $\operatorname{Comp}(R)$ is prescribed. We first show that any group $G$ can appear as $\operatorname{Comp}(R)$ for some skew group ring $R$. We thank M. Beattie for her help on this example.

Example 15. Let $G$ be any group and let $S=k\left[X_{g} \mid g \in G\right]$ be a polynomial ring over a field $k$ in commuting indeterminants indexed by $G$. Note that $G \cong\left\{\phi_{g}\right.$ : $\left.X_{h} \mapsto X_{g h} \mid g \in G\right\} \subset \operatorname{Aut}(S)$. Define $R=S * G$, the skew group ring. Since $R_{g}=S * \phi_{g}$, we see that $R_{g} \cong{ }_{1}(S)_{\phi_{g}}$ as bimodules. But ${ }_{1}(S)_{\phi_{g}} \cong{ }_{1}(S)_{\phi_{h}} \Leftrightarrow g=h$, and so, $G \cong \operatorname{Comp}(R)$.

The preceding example implies, of course, that any abelian group can be realized as the component group of a strongly graded ring. However, D. F. Anderson [1] has 
a nice construction technique for integral domains that are graded by commutative, torsionless, cancellative monoids. Once we put Anderson's result in our perspective, we shall see that not only can we construct a commutative, strongly $G$-graded ring $R$ with a prescribed $\operatorname{Comp}(R)$ group, but we can partially prescribe $\operatorname{Pic}\left(R_{1}\right)$ and $\operatorname{Pic}(R)$ as well.

Proposition 16 (cf. [1, Theorem, p. 249]). Let $R$ be a strongly G-graded, commutative ring. If $\theta: \operatorname{Picent}\left(R_{1}\right) \rightarrow \operatorname{Picent}(R)$ is defined via $I \rightarrow I R$, then $\operatorname{Comp}(R)=\operatorname{ker}(\theta)$.

Proof. (C) If $\left[R_{g}\right] \in \operatorname{Comp}(R)$, then

$$
R_{g} \cdot R=R_{g}\left(\bigoplus_{x \in G} R_{x}\right)=\bigoplus_{x \in G} R_{g x}=R
$$

and so $\left[R_{g}\right] \in \operatorname{ker}(\theta)$.

(つ) Let $[I] \in \operatorname{ker}(\theta)$ so that $I R \cong R$, as bimodules. Since $I R$ is a nonzero, homogeneous, principal ideal, there exists $z \in R_{g}$ for some $g \in G$ such that $I R=z R$. Since we may assume that $I \subset R_{1}$, we see that $I R=\bigoplus_{x \in G} I R_{x}=z R=\bigoplus_{x \in G} z R_{x}$ and so $I=z R_{g^{-1}}$. Consequently, $[I]=\left[R_{g^{-1}}\right]$ as an element in $\operatorname{Comp}(R)$.

Corollary 17 (cf. [1, Example, p. 251]). Given a short exact sequence of abelian groups $1 \rightarrow A \rightarrow B \rightarrow C \rightarrow 1$, there exists a strongly graded, commutative ring $R$ such that $\operatorname{Comp}(R) \cong A, \operatorname{Pic}\left(R_{1}\right) \cong B$, and $\operatorname{Pic}(R) \cong C$.

Proof. By [1, Example, p. 251], there exists a strongly graded commutative ring $R$ such that $\operatorname{Pic} R_{1} \cong B$, Pic $R \cong C$, and $\operatorname{ker} \theta \cong A$. Now apply Proposition 16 .

We close with a final example of a noncommutative strongly graded ring $R$ that is graded equivalent to a crossed product, but has the property that $\operatorname{Comp}(R) \not \subset$ $\operatorname{Pic}_{n}\left(R_{1}\right)$ and $\operatorname{Comp}(R)$ is torsionfree. Moreover, $R_{1}$ is Azumaya. Yet $R$ is graded equivalent to a crossed product by Theorem 3 . This serves to show that Theorem 10 cannot be extended to arbitrary rings without modification. We thank L. Levy for his help on this example.

Example 18. Let $k$ be any field and let $K=k\left(X_{i} \mid i \in \mathbf{Z}\right)$, the rational function field with countably many indeterminants. Define $\sigma \in \operatorname{Aut}(K)$ via the rule $X_{i} \mapsto$ $X_{i+1}$ and extend linearly. It follows that $\langle\sigma\rangle$ is an infinite cyclic subgroup of $\operatorname{Aut}(K)$; let $G=\langle\sigma\rangle$.

Set $T$ to be the following ring and $X$ to be the following $T$-bimodule, both contained in $M_{3}(K)$ :

$$
T=\left(\begin{array}{ccc}
K & K & 0 \\
K & K & 0 \\
0 & 0 & K
\end{array}\right), \quad X=\left(\begin{array}{ccc}
0 & 0 & K \\
0 & 0 & K \\
K & K & 0
\end{array}\right) .
$$

Note that $X \otimes X \cong X \cdot X=T$ and so $X \in \operatorname{Pic}(T)$. Finally, $T$ is a semiperfect, Azumaya algebra.

Set $R$ to be a $\mathbf{Z}$-graded ring defined via $R=\bigoplus_{n \in \mathbf{Z}} R_{n}$ where $R_{n}=X^{n} \cdot \sigma^{n}$. The $T$-bimodule $R_{n}$ inherits the left $T$-multiplication from the left $T$-action on $X$, and the right $T$-action is skewed by $\sigma^{n}: x \cdot t=x\left(\sigma^{n}(t)\right)$. Notice that $\bigotimes^{s} R_{m} \cong R_{s m} \nsucceq T$ and so $\operatorname{Comp}(R)$ is torsionfree. Further, since $X^{(n)} ¥ T^{(n)}$ for any positive integer $n, \operatorname{Comp}(R) \not \subset \mathrm{Pic}_{n}(R)$. Nonetheless, since $T$ is semiperfect, $R$ is graded equivalent 
to a crossed product $S$ by Theorem 3 . In fact, we see that $S_{1}$ is the matrix ring

$$
S_{1}=\left(\begin{array}{cc}
K & 0 \\
0 & K
\end{array}\right)
$$

so that $S=\bigoplus S_{n}$ where

$$
S_{n}=S_{1} \cdot\left(\begin{array}{ll}
0 & 1 \\
1 & 0
\end{array}\right)^{n} \cdot x^{n} .
$$

\section{REFERENCES}

1. D. F. Anderson, The kernel of $\operatorname{Pic}\left(R_{0}\right) \rightarrow \operatorname{Pic}(R)$ for $R$ a graded domain, Mathematical Reports of the Academy of Science, vol. 13, The Royal Society of Canada, 1991, pp. 248-253. MR 92j:13001

2. F. Anderson and K. Fuller, Rings and categories of modules, Springer-Verlag, New York, 1974. MR 54:5281

3. H. Bass, Algebraic K-theory, Benjamin, New York, 1968. MR 40:2736

4. H. Bass and R. Guralnick, Projective modules with free multiples and powers, Proc. Amer. Math. Soc. 96 (1986), 207-208. MR 87a:13011

5. (1988), 213-217. MR 89e: 13006

6. M. Beattie, A generalization of the smash product of a graded ring, J. Pure Appl. Algebra 52 (1988), 219-226. MR 89k:16005

7. M. Cohen and S. Montgomery, Group-graded rings, smash products, and group actions, Trans. Amer. Math. Soc. 282 (1984), 237-258. MR 85i:16002

8. C. Curtis and I. Reiner, Methods of representation theory with applications to finite groups and orders, Vol. 2, Wiley, New York, 1987. MR 88f:20002

9. E. Dade, Group-graded rings and modules, Math. Z. 174 (1980), 241-262. MR 82c:16028

10. R. Guralnick, Bimodules over pi rings, Methods in Module Theory, Marcel Dekker, New York, 1993, pp. 117-134. MR 94a:16001

11. R. Guralnick and S. Montgomery, On invertible bimodules and automorphisms of noncommutative rings, Trans. Amer. Math. Soc. 341 (1994), 917-937. MR 94d:16027

12. R. Gordon and E. Green, Graded Artin algebras, J. Algebra 76 (1982), 111-137. MR 83m:16028a

13. J. Haefner, Graded Morita theory for infinite groups, J. Algebra 169 (1994), 552-586. MR 95j:16051 14. $\frac{}{95: 09}$, Graded equivalence theory with applications, J. Algebra 172 (1995), 385-423. CMP

15. A strongly graded ring that is not graded equivalent to a skew group ring, Comm. Algebra 22 (1994), 4795-4799. MR 95c:16055

16. C. Menini and C. Năstăsescu, When is R-gr equivalent to the category of modules? J. Pure Appl. Algebra 51 (1988), 277-291. MR 89f:16002

17. D. Passman, Infinite crossed products, Academic Press, New York, 1989. MR 90g:16002

18. D. Quinn, Group-graded rings and duality, Trans. Amer. Math. Soc. 292 (1985), 155-167. MR 87d:16002

19. A. del Río, Graded rings and equivalences of categories, Comm. Algebra 19 (1991), 997-1012. MR 92d:16011

Department of Mathematics, University of Colorado, Colorado Springs, Colorado 80933

E-mail address: haefner@math.uccs.edu 